\title{
Trajectory Planning Based on Screw Theory with Consideration of the Optimal Berth Position for a Space Robot
}

\author{
Yong Wang $\mathbb{D}$, Ying Liao $(\mathbb{D}$, and Kejie Gong $(\mathbb{D}$ \\ College of Aerospace Science and Engineering, National University of Defense Technology, No. 109 Deya Road, Changsha, \\ Hunan 410073, China \\ Correspondence should be addressed to Ying Liao; liaoying1104@nudt.edu.cn
}

Received 5 August 2019; Accepted 18 September 2019; Published 29 October 2019

Academic Editor: Paolo Gasbarri

Copyright () 2019 Yong Wang et al. This is an open access article distributed under the Creative Commons Attribution License, which permits unrestricted use, distribution, and reproduction in any medium, provided the original work is properly cited.

\begin{abstract}
Trajectory planning is a prerequisite for the tracking control of a free-floating space robot. There are usually multiple planning objectives, such as the pose of the end-effector and the base attitude. In efforts to achieve these goals, joint variables are often taken as exclusive operable parameters, while the berth position is neglected. This paper provides a novel trajectory planning strategy that considers the berth position by applying screw theory and an optimization method. First, kinematic equations at the position level are established on the basis of the product of exponential formula and the conservation of the linear momentum of the system. Then, generalized Jacobian matrices of the base and end-effector are derived separately. According to the differential relationship, an ordinary differential equation for the base attitude is established, and it is solved by the modified Euler method. With these sufficient and necessary preconditions, a parametric optimization strategy is proposed for two trajectory planning cases: zero attitude disturbance and attitude adjustment of the base. First, the berth position is transformed into the desired position of the end-effector, and its constraints are described. Joint variables are parameterized using a sinusoidal function combined with a five-order polynomial function. Then, objective functions are constructed. Finally, a genetic algorithm with a modified mutation operator is used to solve this optimization problem. The optimal berth position and optimized trajectory are obtained synchronously. The simulation of a planar dual-link space robot demonstrates that the proposed strategy is feasible, concise, and efficient.
\end{abstract}

\section{Introduction}

On-orbit servicing (OOS) [1] technology has been attracting increasing research and investment from space institutions worldwide for its potential to mitigate on-orbit failures [2] and maintain and extend outer-space projects. Space robots are one of the most successful and extensively used means to achieve these goals, as demonstrated by several notable deployments and experimental missions, such as Space Station Remote Manipulator System (SSRMS) [3], Experimental Test Satellite VII (ETS-VII) [4], Front-end Robotics Enabling Near-term Demonstration (FREND) [5], and China's intelligent space robots [6]. The concept of cooperative small robotic servicers to handle large passive objects has also been studied in recent years [7-9]. Possible applications of space robots include inspection, assembly, maintenance, repair, and refueling [10]. When executing these tasks, the system often works in one of three modes: free-flying mode (in which the position and attitude of the base are both controllable) [11], free-translation mode (in which only the base attitude is controllable), and free-floating mode [12] (in which the base is completely free).

Trajectory planning-a term that is often used interchangeably with path planning, despite their technical differences-is crucial for implementing a space mission. Many research papers have addressed the trajectory planning and control issues of the free-floating space robot because it consumes relatively little fuel and avoids potential collisions that can result from excess control [13]. The distinctive feature of a free-floating space robotic system is that it is nonholonomic because the angular momentum equation cannot be integrated. This feature has been used to develop some helpful planning strategies, known as nonholonomic trajectory planning. Vafa and Dubowsky [14] proposed the self-correcting 
motion method, which adjusts the base attitude by adding small cyclic motions to the joint motions. They also developed another method called the Disturbance Map (DM) [15]. The DM method builds a relationship between each point in the joint space and the actual movement of the base, and then the trajectory of the joints that causes the minimum disturbance to the base can be selected. Dubowsky and Torres $[16,17]$ improved the DM method to minimize spacecraft attitude disturbance. They called the improved method the Enhanced Disturbance Map (EDM), which can also be used to minimize the fuel consumption for attitude control. Fernandes et al. [18] were inspired by the falling cat problem and developed a near-optimal motion planning method. Papadopoulos et al. $[19,20]$ proposed a path planning methodology that allows for simultaneous control of the endeffector and the base attitude. In this approach, polynomials are employed to obtain a smooth and continuous trajectory. $\mathrm{Xu}$ et al. [21] developed a coordinated trajectory planning method to stabilize the base attitude and centroid position by a balance arm.

In recent years, optimization algorithms have been introduced in studies that aim to achieve optimal trajectories for free-floating space robots. Huang et al. [22] provided an approach based on a Genetic Algorithm (GA) to find a global minimum-jerk trajectory of a space manipulator in the joint space. Xu et al. [23] employed sinusoidal functions, whose arguments are seven-order polynomials, and a GA to realize the base attitude and attain the desired states of the joints. They also proposed a similar planning strategy in Cartesian space and searched for the optimal parameters using the Particle Swarm Optimization (PSO) algorithm [24]. PSO was also used in the studies in [25, 26]. Rybus and Seweryn [27] employed the Rapidly-exploring Random Trees (RRT) algorithm for the trajectory planning of a satellite-manipulator system, and joint limits and obstacles were taken into account. Luo et al. [28] proposed a novel trajectory planning framework with a task-priority handling strategy for a space robot. A Quadratic Programming (QP) procedure was applied to solve the problem.

In the above studies, the parameterization and optimization approaches have been successfully applied to solve trajectory planning problems. Nevertheless, the parameters to be optimized are often exclusively chosen as coefficients of joint trajectories that are represented by continuous functions. Before executing the mission, the base (spacecraft) hovers at a proper position (called the berth position) relative to the target to avoid unexpected collision and change in the work mode. The variation in the berth position markedly affects the desired trajectories of the joints; however, it seems to have been frequently omitted from trajectory planning studies. Zhang and Liu [29] provided a new viewpoint that utilizes the base berth position as an optimizable parameter. A unified motion planning strategy based on the forward kinematics and parametrical optimization method was developed. The objective function was simplified by proposing the concept of grasping area and using a penalty function. Finally, PSO was employed to determine the parameters. It was demonstrated that the DOF requirement of the manipulator could be reduced by taking the berth position into account. This innovative idea has great potential to diversify the objectives of a single mission. However, only the desired position and attitude of the end-effector are satisfied. Furthermore, the berth position is not optimized directly; instead, it is calculated from the optimal results of the joint variables according to a geometric relationship. This point is worth further exploring.

Another point of current research that can be expanded is the kinematical theory. Kinematic equations constitute the objective function, which directly affects the efficiency of a given trajectory planning strategy. Because space robots differ from ground-based robots, researchers have developed new kinematical theories for space robots, including the Virtual Manipulator (VM) method [14], Generalized Jacobian Matrix (GJM) [30], and Dynamically Equivalent Manipulator (DEM) [31]. GJM is widely used in control of space robots $[32,33]$. However, when applied to trajectory planning, the VM and DEM methods focus on the transformation of the model, and the transformation process significantly increases the workload. Furthermore, deriving the GJM with body-fixed vectors makes the programming difficult. Besides, integration is needed when calculating the displacement of joints and the end-effector at the position level, as Zhang and Liu did in the autonomous trajectory planner [34].

Screw theory is an efficient alternative to the kinematic analysis of a rigid body and its mechanism. It can establish a global description of the rigid body and avoid singularities [35]. It has been applied to research on the ground-based robot [36] and parallel manipulator $[37,38]$. The unified description of translation and rotation confers the advantages of conciseness and efficiency to screw theory. Rocha et al. [39] compared the screw-based method with the Denavit-Hartenberg method [40] in detail and concluded that the former has advantages over the $\mathrm{D}-\mathrm{H}$ approach, although the latter is more commonly used. Nevertheless, few studies on space robots using screw theory are available in the current literature. Liu and $\mathrm{Wu}$ [41] developed a dynamic model of a space robot system on the basis of screw theory and Kane's equation. Liu and $\mathrm{Wu}$ [42] provided a simple and efficient method to derive the Jacobian matrix of a free-floating branching robot system by dividing it into two parts: the ground-robot with a fixed base and a locked-joint robot with a floating base. The authors of this paper studied kinematic problems at position level base on screw theory [43]; a part of these works will be applied in this research.

This paper proposes a novel trajectory planning strategy based on screw theory. The proposed approach considers the berth position and aims to improve calculation efficiency. First, the kinematic model of the free-floating space robot is built using screw theory, and the model includes the position and differential level. The base attitude is calculated using the modified Euler method. Next, we construct the optimization problem by transforming the berth position and parameterizing the joint variables. Then, the planning strategy is provided in detail. A simulation of a planar dual-link space robot is presented, and Adams simulation results are provided to verify the proposed method. A nomenclature is given to clarify important variables and abbreviations. 


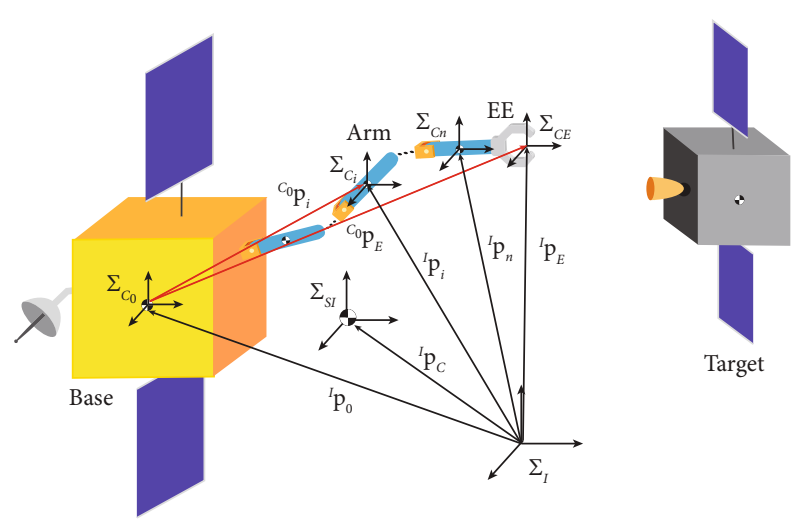

Figure 1: Model of the space robot system.

\section{Kinematic Modeling Using Screw Theory}

2.1. Model of the Space Robotic System. A space robot (also referred to as a space manipulator) is a very complicated system, which can be simplified if only kinematics is considered. Figure 1 provides a general model of the space robotic system, which consists of the base (spacecraft), $\operatorname{arm}(\mathrm{s})$, and the End-Effector (EE). The arm contains several links and joints. The following assumptions are made to build a concise and accurate kinematic model.

1. The position and orientation of the base is not controlled, and there are no external forces applied to the system, which means that the system is in freefloating mode

2. The initial linear and angular momentum of the system are both zero

3. The arm is a single chain connected to the base, and the parallel manipulator is not considered

4. All links and joints are rigid

5. All joints are revolute, and all rotation angles are limited between $-180^{\circ}$ and $180^{\circ}$

2.2. Kinematic Equations at the Position Level. According to the product of exponentials (POE) formula, the final pose of a centroid-fixed frame of the $i^{\text {th }}$ link with respect to the base-fixed frame can be defined as follows:

$$
\begin{aligned}
{ }_{C_{i}}^{C_{0}} g & =e^{\theta_{1} \widehat{\xi}_{1}} e^{\theta_{2} \hat{\xi}_{2}} \cdots e^{\theta_{i} \widehat{\xi}_{i} C_{0}} g(0) \\
& =\left[\begin{array}{cc}
{ }^{C_{0}} R_{i} & { }^{C_{0}} \mathbf{p}_{i} \\
0 & 1
\end{array}\right], \quad i=1,2, \cdots, n,
\end{aligned}
$$

where $\xi_{i}=\left(\omega_{i}, r_{i} \times \omega_{i}\right)^{\mathrm{T}}$ denotes the twist coordinates of $\widehat{\xi}_{i}$.

Then, the final pose of the end-effector with respect to the base-fixed frame can be determined by

$$
{ }_{E}^{C_{0}} \mathfrak{g}=e^{\theta_{1} \hat{\xi}_{1}} e^{\theta_{2} \hat{\xi}_{2}} \cdots e^{\theta_{n} \hat{\xi}_{n} C_{0}} g(0)=\left[\begin{array}{cc}
{ }^{C_{0}} R_{E} & { }^{C_{0}} \mathbf{p}_{E} \\
0 & 1
\end{array}\right]
$$

The final pose of the base and the centroid-fixed frame of the $i^{\text {th }}$ link with respect to the inertial frame can be expressed as follows:

$$
{ }_{C_{i}}^{I} g=\left[\begin{array}{cc}
{ }^{I} R_{i} & { }^{I} \mathbf{p}_{i} \\
0 & 1
\end{array}\right], \quad i=0,1, \cdots, n,
$$

where ${ }^{I} R_{0}$ is determined by the orientation of the base. If we define the attitude angles (roll, pitch, yaw) of the base as $\Psi=(\delta, \beta, \gamma)$, then

$$
{ }^{I} R_{0}=\left[\begin{array}{ccc}
c \beta c \gamma & s \delta s \beta c \gamma-c \delta s \gamma & c \delta s \beta c \gamma+s \delta s \gamma \\
c \beta s \gamma & s \delta s \beta s \gamma+c \delta c \gamma & c \delta s \beta s \gamma-s \delta c \gamma \\
-s \beta & s \delta c \beta & c \delta c \beta
\end{array}\right],
$$

where $c$ denotes cos and $s$ denotes sin.

In terms of the transformation rule of rigid motion, the following equation holds:

$$
\begin{aligned}
{ }_{C_{i}}^{I} g & ={ }_{C_{0}}^{I} g_{C_{i} C_{0}} g=\left[\begin{array}{cc}
{ }^{I} R_{0} & { }^{I} \mathbf{p}_{0} \\
0 & 1
\end{array}\right]\left[\begin{array}{cc}
{ }^{C_{0}} R_{i} & { }^{C_{0}} \mathbf{p}_{i} \\
0 & 1
\end{array}\right] \\
& =\left[\begin{array}{cc}
{ }^{I} R_{0}{ }^{C_{0}} R_{i} & { }^{I} R_{0}{ }^{C_{0}} \mathbf{p}_{i}+{ }^{I} \mathbf{p}_{0} \\
0 & 1
\end{array}\right] .
\end{aligned}
$$

Thus,

$$
\left\{\begin{array}{l}
{ }^{I} R_{i}={ }^{I} R_{0}{ }^{C_{0}} R_{i} \\
{ }^{I} \mathbf{p}_{i}={ }^{I} R_{0}{ }^{C_{0}} \mathbf{p}_{i}+{ }^{I} \mathbf{p}_{0}
\end{array}\right.
$$

Then, the final pose of the end-effector with respect to the inertial frame can be derived as follows:

$$
\begin{aligned}
& { }_{E}^{I} \mathfrak{g}={ }_{C_{0}}^{I} g_{E}^{C_{0}} \mathfrak{g}=\left[\begin{array}{cc}
{ }^{I} R_{0} & { }^{I} \mathbf{p}_{0} \\
0 & 1
\end{array}\right]\left[\begin{array}{cc}
{ }^{C_{0}} R_{E} & { }^{C_{0}} \mathbf{p}_{E} \\
0 & 1
\end{array}\right] \\
& =\left[\begin{array}{cc}
{ }^{I} R_{0}{ }^{C_{0}} R_{E} & { }^{I} R_{0}{ }^{C_{0}} \mathbf{p}_{E}+{ }^{I} \mathbf{p}_{0} \\
0 & 1
\end{array}\right] \text {. }
\end{aligned}
$$

In the inertial frame, the centroid formula of the system is written as follows:

$$
m_{0}{ }^{I} \mathbf{p}_{0}+\sum_{i=1}^{n} m_{i}^{I} \mathbf{p}_{i}=M^{I} \mathbf{p}_{C}
$$

Substituting Equation (6) into Equation (8) yields

$$
{ }^{I} \mathbf{p}_{0}={ }^{I} \mathbf{p}_{C}-\frac{{ }^{I} R_{0}}{M} \sum_{i=1}^{n} m{ }_{i}{ }^{C} \mathbf{p}_{i}={ }^{I} \mathbf{p}_{C}-{ }^{I} R_{0} \mathbf{p}_{M},
$$

where $\mathbf{p}_{M}=1 / M \sum_{i=0}^{n} m_{i}{ }^{C} \mathbf{p}_{i}$. In particular, ${ }^{I} \mathbf{p}_{C}=0$ when the centroid of the system is chosen as the initial point of the inertial frame. 
Substituting Equation (9) into Equation (7) yields

${ }_{E}^{I} \boldsymbol{g}=\left[\begin{array}{cc}{ }^{I} R_{0}{ }^{C_{0}} R_{E} & { }^{I} R_{0}\left({ }^{C_{0}} \mathbf{p}_{E}-\mathbf{p}_{M}\right)+{ }^{I} \mathbf{p}_{C} \\ 0 & 1\end{array}\right], \quad i=1,2, \cdots, n$.

2.3. The Generalized Jacobian Matrix. Angular momentum of the base and the $i^{\text {th }}$ link with respect to the inertial frame can be defined as follows:

$$
L_{i}=I_{C_{i}} \boldsymbol{\omega}_{i}^{B}+m_{i}^{I} \mathbf{p}_{i} \times{ }^{I} \dot{\mathbf{p}}_{i},
$$

where $\boldsymbol{\omega}_{i}^{B}$ is the body angular velocity of the $i^{\text {th }}$ link. The body velocity of the base and the $i^{\text {th }}$ link is defined as follows:

$$
{ }_{C_{i}}^{I} V^{B}=\left({ }_{C_{i}}^{I} g^{-1}{ }_{C_{i}} \dot{g}\right)=\left[\begin{array}{c}
{ }^{I} \boldsymbol{\omega}_{i}^{B} \\
{ }^{I} \mathbf{v}_{i}^{B}
\end{array}\right]=\left[\begin{array}{c}
\left({ }^{I} R_{i}^{{ }^{T} I} \dot{R}_{i}\right)^{\mathrm{V}} \\
{ }^{{ }^{I}} R_{i}^{T_{I}} \dot{\mathbf{p}}_{i}
\end{array}\right] .
$$

Similarly, the spatial velocity is specified as follows:

$$
{ }_{C_{i}}^{I} V^{S}=\left({ }_{{ }_{i}}^{I} \dot{g}_{C_{i}}^{I} g^{-1}\right)^{\mathrm{V}}=\left[\begin{array}{c}
{ }^{I} \boldsymbol{\omega}_{i}^{S} \\
{ }^{I} \mathbf{v}_{i}^{S}
\end{array}\right]=\left[\begin{array}{c}
\left({ }^{I} \dot{R}_{i}^{I} R_{i}^{T}\right)^{\mathrm{V}} \\
{ }^{I} \dot{R}_{i}^{I} R_{i}^{T I} \mathbf{p}_{i}+{ }^{I} \dot{\mathbf{p}}_{i}
\end{array}\right] .
$$

The similarity transformation relationship between the body and spatial velocity is specified as follows:

$$
{ }_{C_{i}}^{I} V^{S}=A \underset{C_{i}}{A d_{I}}{ }^{I} C_{i} V^{B} \text { or }_{C_{i}}^{I} V^{B}=A \underset{C_{i}}{A d_{I}} g^{-1} C_{i}^{I} V^{S},
$$

where $A d_{C_{i}} g$ corresponds to the adjoint transformation of ${ }_{C_{i}}^{I} g$, and

$$
A d_{C_{i}} g=\left[\begin{array}{cc}
{ }_{C_{i}}^{I} R & 0 \\
{ }^{I} \widehat{\mathbf{p}}_{i C_{i}}^{I} R & { }_{C_{i}}^{I} R
\end{array}\right]
$$

Then, $L_{i}$ can be derived as follows:

$$
\begin{aligned}
L_{i} & =\left[I_{C_{i}} \nabla m_{i}{ }^{I} \widehat{\mathbf{p}}_{i}{ }^{I} R_{i}\right]\left[\begin{array}{c}
{ }^{I} \omega_{i}^{B} \\
{ }^{I} R_{i}^{T} I_{\mathbf{p}_{i}}
\end{array}\right] \\
& =\left[\begin{array}{ll}
I_{C_{i}} & m_{i}{ }_{i} \widehat{\mathbf{p}}_{i}{ }^{I} R_{i}
\end{array}\right] A d_{{ }^{I}{ }_{i}} g^{-1} C_{i} V^{S} \\
& =\left[\begin{array}{ll}
I_{C_{i}}{ }^{I} R_{i}^{T}-m_{i}{ }^{I} \widehat{\mathbf{p}}_{i}{ }^{I} \widehat{\mathbf{p}}_{i} & m_{i}{ }^{I} \widehat{\mathbf{p}}_{i}
\end{array}\right]^{I} C_{i} V^{S}=K_{i C_{i}}^{I} V^{S},
\end{aligned}
$$

where $K_{i}\left[I_{C_{i}}^{I} R_{i}^{T}-m_{i}^{I} \widehat{\mathbf{p}}_{i}^{I} \widehat{\mathbf{p}}_{i} \quad m_{i}^{I} \widehat{\mathbf{p}}_{i}\right]$, which can be defined as the generalized inertia matrix.
In terms of the transformation rule of spatial velocities, the following equation holds:

$$
{ }_{C_{i}}^{I} V^{S}={ }_{C_{0}}^{I} V^{S}+A \underset{C_{0} g}{ }{ }^{C_{0}} C_{i}^{S}
$$

From Equations (16) and (17), the angular momentum of the system can be defined by

$L=\sum_{i=1}^{n} K_{i}\left({ }_{C_{0}}^{I} V^{S}+\underset{C_{0}}{A d_{I}}{ }^{C_{0}} C_{i}^{S}\right)+K_{0 C_{0}}^{I} V^{S}=\tilde{K}_{B C_{0}}^{I} V^{S}+\tilde{K}_{N} J_{N} \dot{\boldsymbol{\theta}}$,

where $\quad \tilde{K}_{B}=\sum_{i=0}^{n} K_{i} \in \mathbb{R}^{3 \times 6}, \quad \tilde{K}_{N}=$ $\left[\begin{array}{cccc}K_{1} A d_{C_{0}} g & K_{2} A d_{C_{0} I_{g}} & \cdots & K_{n} A d_{C_{0}} g\end{array}\right] \in \mathbb{R}^{3 \times 6 n}$, and $J_{N}=$ $\left[\begin{array}{llll}J_{1} & J_{2} & \cdots & J_{n}\end{array}\right]^{\mathrm{T}} \in \mathbb{R}^{6 n \times n}, \quad J_{i}=\left(\widehat{\boldsymbol{\xi}}_{1}{ }^{\prime}, \widehat{\boldsymbol{\xi}}_{2}{ }^{\prime}, \cdots \widehat{\boldsymbol{\xi}}_{i}{ }^{\prime}, 0, \cdots 0\right)^{\mathrm{T}} \epsilon$ $\mathbb{R}^{n \times 6}$.

The angular momentum of the system is conserved. Without loss of generality, it is considered to be zero. Thus,

$$
{ }_{C_{0}}^{I} V^{S}=-\tilde{K}_{B}^{+} \tilde{K}_{N} J_{N} \dot{\boldsymbol{\theta}}=J_{B} \dot{\boldsymbol{\theta}}
$$

where $\tilde{K}_{B}^{+}$is the pseudoinverse matrix of $\tilde{K}_{B} \cdot J_{B}=-\tilde{K}_{B}^{+}$ $\tilde{K}_{N} J_{N}$, which is defined as the generalized Jacobian matrix of the base.

Furthermore,

$$
{ }_{E}^{I} V^{S}={ }_{C_{0}}^{I} V^{S}+A d_{C_{0}} g E{ }^{C_{0}} V^{S}=\left(J_{B}+A d_{C_{0}} g J_{n}\right) \dot{\boldsymbol{\theta}}=J_{E} \dot{\boldsymbol{\theta}},
$$

where $J_{E}=\left(J_{B}+A d_{C_{0}} g J_{n}\right)$, which is defined as the generalized Jacobian matrix of the end-effector.

2.4. Attitude Calculation of the Base Using the Generalized Jacobian Matrix. Equation (19) can be rewritten as follows:

$$
{ }_{C_{0}}^{I} V^{S}=\left[\begin{array}{c}
{ }^{I} \boldsymbol{\omega}_{0}^{S} \\
I_{\mathbf{v}_{0}^{S}}^{S}
\end{array}\right]=J_{B} \dot{\boldsymbol{\theta}}=\left[\begin{array}{c}
J_{B \omega} \\
J_{B v}
\end{array}\right] \dot{\boldsymbol{\theta}}
$$

where $J_{B \omega}, J_{B v} \in \mathbb{R}^{3 \times n}$ is the submatrix of $J_{B}$ related to orientation and position, respectively. According to the deductions in Subsections 2.2 and 2.3, it is apparent that $J_{B \omega}$ is a function that depends on $\Psi$. Thus,

$$
{ }^{I} \boldsymbol{\omega}_{0}^{S}=J_{B \omega}(\Psi) \dot{\boldsymbol{\theta}}
$$

On the other hand, according to Equations (4) and (13),

$$
{ }^{I} \boldsymbol{\omega}_{0}^{S}=\left({ }^{I} \dot{R}_{0}{ }^{I} R_{0}^{T}\right)^{\mathrm{V}}=\left[\begin{array}{ccc}
0 & -s \gamma & c \gamma c \beta \\
0 & c \gamma & s \gamma c \beta \\
1 & 0 & -s \beta
\end{array}\right]\left[\begin{array}{c}
\dot{\delta} \\
\dot{\beta} \\
\dot{\gamma}
\end{array}\right]=J_{\text {Euler }}(\Psi) \Psi
$$


where

$$
J_{\text {Euler }}=\left[\begin{array}{ccc}
0 & -s \gamma & c \gamma c \beta \\
0 & c \gamma & s \gamma c \beta \\
1 & 0 & -s \beta
\end{array}\right] .
$$

Substituting Equation (23) into Equation (22) yields

$$
\dot{\Psi}=J_{\text {Euler }}^{-1}(\Psi) J_{B \omega}(\Psi) \dot{\boldsymbol{\theta}}=F(\Psi, \dot{\boldsymbol{\theta}}, t) .
$$

If $\dot{\boldsymbol{\theta}}$ is given, then Equation (25) is an ordinary differential equation about $\Psi$, which can be solved by the modified Euler method. If the initial state of $\boldsymbol{\Psi}\left(\boldsymbol{\Psi}\left(t_{0}\right)\right)$ is known, then

$$
\left\{\begin{array}{l}
\overline{\boldsymbol{\Psi}}_{k}=\boldsymbol{\Psi}_{k-1}+h F\left(\boldsymbol{\Psi}_{k-1}, \dot{\boldsymbol{\theta}}_{k-1}, t_{k-1}\right), \\
\boldsymbol{\Psi}_{k}=\boldsymbol{\Psi}_{k-1}+\frac{h}{2}\left[F\left(\boldsymbol{\Psi}_{k-1}, \dot{\boldsymbol{\theta}}_{k-1}, t_{k-1}\right)+F\left(\overline{\boldsymbol{\Psi}}_{k}, \dot{\boldsymbol{\theta}}_{k}, t_{k}\right)\right], \\
\boldsymbol{\Psi}_{0}=\boldsymbol{\Psi}\left(t_{0}\right), \dot{\boldsymbol{\theta}}_{k}=\dot{\boldsymbol{\theta}}\left(t_{k}\right),
\end{array}\right.
$$

where $h$ is the time step.

\section{Problem Construction}

By considering berth position, we can achieve an extra goal while the EE reaches the desired pose. A relatively simple case is planning a trajectory with zero attitude disturbance to the base. Another case is the adjustment of the attitude of the base to the desired orientation simultaneously. Both trajectory planning problems can be converted to parametric optimization problems. The problems can be described clearly by first performing the following preparations.

3.1. Transformation of the Berth Position. The problem can be simplified by disregarding the movement of the target. Thus, the berth position can be directly expressed as the vector ${ }^{I} \mathbf{p}_{t b}$ from the target's centroid to the base's centroid, which is the variable we aim to optimize. With respect to the target-centroid-fixed inertial frame $\sum_{T I}$, the desired position of the end-effector ${ }^{I} \mathbf{p}_{t e}$ is constant, while the centroid of the system varies with ${ }^{I} \mathbf{p}_{t b}$ because of the conservation of linear momentum. Conversely, the problems become complex if they are analyzed in $\sum_{T I}$. Given that the berth position has no effect on the kinematic relationship, and the centroid of the system will be immobile once the berth position is determined, kinematic equations can be made much more concise when expressed in the system-centroid-fixed inertial frame $\sum_{S I}$. In fact, if the berth position is expressed with respect to $\sum_{S I}$, then the kinematic equations contain no variables of the berth position. Only the target's centroid, as well as the desired position of the EE, varies with ${ }^{I} \mathbf{p}_{t b}$, which means that the berth position is transformed into the desired position of the EE. The transformation process is shown in Figure 2. It must be clarified that the transformation is per-

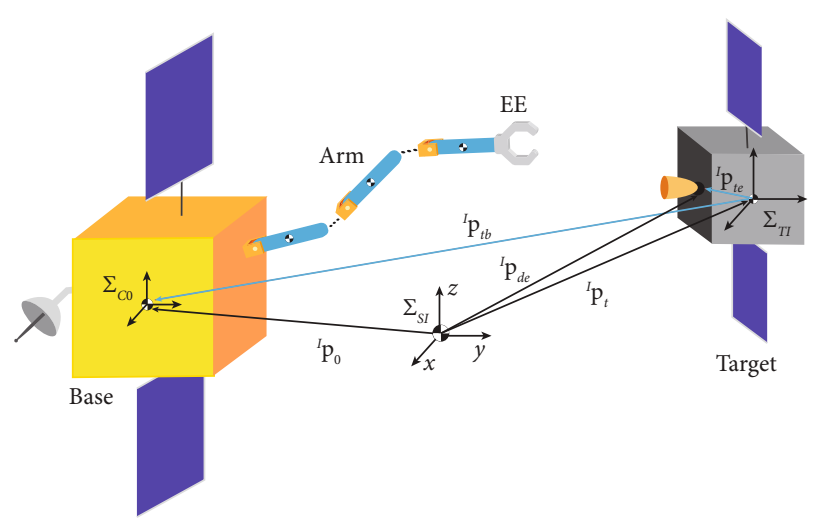

Figure 2: Transformation of the berth position.

formed at the initial time, and all the involved vectors are independent of time.

According to Equation (9), the position of the base's centroid with respect to $\sum_{S I}$ is as follows:

$$
{ }^{I} \mathbf{p}_{0}(0)=-\mathbf{p}_{M}(0)=-\frac{1}{M} \sum_{j=0}^{n} m_{j}{ }^{C_{0}} \mathbf{p}_{j}(0)
$$

Then, the position of the target's centroid with respect to $\sum_{S I}$ can be derived as follows:

$$
{ }^{I} \mathbf{p}_{t}={ }^{I} \mathbf{p}_{0}(0)-{ }^{I} \mathbf{p}_{t b}=-\mathbf{p}_{M}(0)-{ }^{I} \mathbf{p}_{t B} .
$$

Then, the desired position of the EE with respect to $\sum_{S I}$ can be derived as follows:

$$
{ }^{I} \mathbf{p}_{d e}={ }^{I} \mathbf{p}_{t}+{ }^{I} \mathbf{p}_{t e}={ }^{I} \mathbf{p}_{t e}-\mathbf{p}_{M}(0)-{ }^{I} \mathbf{p}_{t b} .
$$

Further, the desired orientation of the EE should also be transformed:

$$
{ }_{E}^{I} R_{d}={ }_{T}^{I} R_{P}^{T} R_{E}^{P} R_{d}
$$

where ${ }_{E}^{P} R_{d}$ is the desired rotation matrix from the capture point to the EE, ${ }_{P}^{T} R$ is the rotation matrix of the capture point with respect to $\sum_{T I}$, and ${ }_{T}^{I} R$ is the rotation matrix of $\sum_{T I}$ with respect to $\sum_{S I}$.

Next, constraints of ${ }^{I} \mathbf{p}_{t b}$ need to be specified.

(1) Safe distance, which is designed to avoid unexpected collision while the space robot system is docking, is expressed as follows:

$$
\left\|{ }^{I} \mathbf{p}_{t b}\right\|>P_{s}
$$

where $P_{s}$ is the threshold value of safe distance.

(2) In workspace, which is designed to guarantee that the capture point is reachable by the EE, is expressed as 
follows:

$$
\left\|{ }^{I} \mathbf{p}_{d e}\right\|<R_{w}
$$

where $R_{w}$ is the radius of the system's guaranteed workspace. In terms of the inequality property of the norm,

$$
\left\|{ }^{I} \mathbf{p}_{t b}\right\|=\left\|{ }^{I} \mathbf{p}_{t e}-\mathbf{p}_{M}(0)-{ }^{I} \mathbf{p}_{d e}\right\|<R_{w}+\left\|{ }^{I} \mathbf{p}_{t e}\right\|+\left\|\mathbf{p}_{M}(0)\right\| .
$$

(3) Proper location, which is designed to prevent the system from docking at the opposite side of the capture point. Considering the situation in Figure 2,

$$
{ }^{I} \mathbf{p}_{t b x}<{ }^{I} \mathbf{p}_{t e x},
$$

where ${ }^{I} \mathbf{p}_{t b x}$ and ${ }^{I} \mathbf{p}_{t e x}$ are projections of ${ }^{I} \mathbf{p}_{t b}$ and ${ }^{I} \mathbf{p}_{t e}$ along the $x$ axis, respectively.

3.2. Parameterization of Joint Variables. Trajectory planning in the joint space requires that the joint variables be parameterized first. Many methods have been developed to obtain a feasible and smooth trajectory. Since these variables are bounded, we chose a concise and effective approach-a sinusoidal function combined with five-order polynomial functions-to parameterize the joint angles, angle velocities, and angular accelerations.

$$
\left\{\begin{array}{l}
\theta_{i}=l_{i 1} \sin (A)+l_{i 2}, \\
\dot{\theta}_{i}=l_{i 1} \cos (A) \cdot \dot{A}, \\
\ddot{\theta}_{i}=-l_{i 1} \sin (A) \cdot \dot{A}^{2}+l_{i 1} \cos (A) \cdot \ddot{A},
\end{array}\right.
$$

where $A=a_{i 5} t^{5}+a_{i 4} t^{4}+a_{i 3} t^{3}+a_{i 2} t^{2}+a_{i 1} t+a_{i 0}$, and $l_{i 1}, l_{i 2}$, $a_{i j}(j=0,1, \cdots, 5)$ are undetermined coefficients.

Furthermore, the constraints of these variables are specified as follows.

(1) Equality constraints, including the initial and final states of the joint angles, angle velocities, and angle accelerations, are defined by

$$
\left\{\begin{array}{l}
\boldsymbol{\theta}(0)=0, \\
\dot{\boldsymbol{\theta}}(0)=\dot{\boldsymbol{\theta}}\left(t_{f}\right)=0, \\
\ddot{\boldsymbol{\theta}}(0)=\ddot{\boldsymbol{\theta}}\left(t_{f}\right)=0,
\end{array}\right.
$$

where $t_{f}$ is the final time. Substituting Equation (35) into Equation (36) yields

$$
\left\{\begin{array}{l}
a_{i 0}=-\arcsin \left(\frac{l_{i 2}}{l_{i 1}}\right), \\
a_{i 1}=a_{i 2}=0, \\
a_{i 3}=\frac{5}{3 t_{f}^{2} a_{i 5}}, \\
a_{i 4}=\frac{-5}{2 t_{f} a_{i 5}} .
\end{array}\right.
$$

Then, Equation (35) can be rewritten as follows:

$$
\left\{\begin{array}{l}
\theta_{i}=l_{i 1} \sin (\tilde{A})+l_{i 2}, \\
\dot{\theta}_{i}=l_{i 1} \cos (\tilde{A}) \cdot \dot{\tilde{A}} \\
\ddot{\theta}_{i}=-l_{i 1} \sin (\tilde{A}) \cdot(\dot{\tilde{A}})^{2}+l_{i 1} \cos (\tilde{A}) \cdot \ddot{\tilde{A}}
\end{array}\right.
$$

where $\tilde{A}=a_{i 5}\left(t^{5}-5 / 2 t_{f} t^{4}+5 / 3 t_{f}^{2} t^{3}\right)-\arcsin \left(l_{i 2} / l_{i 1}\right)$.

(2) Inequality constraints, including limits of joint angles, angle velocities, and angular accelerations, are expressed as follows:

$$
\begin{aligned}
& \boldsymbol{\theta}_{\min } \leq \boldsymbol{\theta} \leq \boldsymbol{\theta} \max . \\
& \dot{\boldsymbol{\theta}}_{\min } \leq \dot{\boldsymbol{\theta}} \leq \dot{\boldsymbol{\theta}}_{\max } . \\
& \ddot{\boldsymbol{\theta}}_{\min } \leq \ddot{\boldsymbol{\theta}} \leq \ddot{\boldsymbol{\theta}}_{\max } .
\end{aligned}
$$

According to Equations (38) and (39), $l_{i 1}$ and $l_{i 2}$ can be determined as follows:

$$
\begin{aligned}
& l_{1}=\frac{\boldsymbol{\theta}_{\min }+\boldsymbol{\theta}_{\max }}{2}, \\
& l_{2}=\frac{\boldsymbol{\theta}_{\max }+\boldsymbol{\theta}_{\min }}{2},
\end{aligned}
$$

where $l_{1}=\left\{l_{i 1}\right\}, l_{2}=\left\{l_{i 2}\right\}$.

According to Equations (38), (40), and (41), the boundary limits of $a_{i 5}$ can be determined by

$$
\begin{aligned}
& a_{i 5 \min }=\max \left\{\frac{\dot{\theta}_{i \min }}{l_{i 1} M_{1}}, \frac{\ddot{\theta}_{i \min }}{l_{i 1}\left(M_{1}^{2}+M_{2}\right)}\right\}, \\
& a_{i 5 \max }=\min \left\{\frac{\dot{\theta}_{i \max }}{l_{i 1} M_{1}}, \frac{\ddot{\theta}_{i \max }}{l_{i 1}\left(M_{1}^{2}+M_{2}\right)}\right\},
\end{aligned}
$$

where $M_{1}=\max \left\{\left(5 t^{4}-10 t_{f} t^{3}+5 t_{f}^{2} t^{2}\right), t \in\left(0, t_{f}\right)\right\}$, and $M_{2}$ $=\max \left\{\left(20 t^{3}-30 t_{f} t^{2}+10 t_{f}^{2} t\right), t \in\left(0, t_{f}\right)\right\}$. Let $a_{5}=\left\{a_{i 5}\right\}$, $a_{5 \min }=\left\{a_{i 5 \min }\right\}, a_{5 \max }=\left\{a_{i 5 \max }\right\}$. 
3.3. Objective Function. According to Subsection 3.1, the desired pose of the EE can be determined by

$$
{ }_{E}^{I} g_{d}=\left[\begin{array}{cc}
{ }_{E}^{I} R_{d} & { }^{I} \mathbf{p}_{d e} \\
0 & 1
\end{array}\right]
$$

For the trajectory planning of zero attitude disturbance to the base, the following equality should be satisfied:

$$
{ }_{C_{0}}^{I} \boldsymbol{\omega}^{S}=J_{B \omega} \dot{\boldsymbol{\theta}} \equiv 0
$$

Thus, the objective function for this case can be defined as follows:

$$
J_{z d}=\left\|\log \left({ }_{E}^{I} g_{d}^{-1} I_{E} g\right)\right\|+\int_{0}^{t_{f}}\left\|J_{B \omega} \dot{\boldsymbol{\theta}}\right\| d t
$$

where $\log ()$ means logarithm mapping of a matrix.

For the trajectory planning of the attitude adjustment of the base, the following equality should be satisfied:

$$
\Psi_{f}=\Psi_{d}
$$

Thus, the objective function for this case can be defined as follows:

$$
J_{\alpha \alpha}=\left\|\log \left({ }_{E}^{I} g_{d}^{-1}{ }_{E} g\right)\right\|+\left\|\Psi_{f}-\Psi_{d}\right\|
$$

\section{Trajectory Planning Strategy with Consideration of the Optimal Berth Position}

With the conclusion of Section 3, trajectory planning problems using a parametric optimization method can be described as follows:

$$
\begin{array}{ll}
\min & J_{z d}\left(\boldsymbol{\alpha}_{5},{ }^{I} \mathbf{p}_{t b}\right) \text { or } J_{\alpha \alpha}\left(\boldsymbol{\alpha}_{5},{ }^{I} \mathbf{p}_{t b}\right) \\
\text { st. } & \boldsymbol{\alpha}_{5 \min } \leq \boldsymbol{\alpha}_{5} \leq \boldsymbol{\alpha}_{5 \max } \\
& P_{s}<\left\|{ }^{I} \mathbf{p}_{t b}\right\|<R_{w}+\left\|{ }^{I} \mathbf{p}_{t e}\right\|+\left\|\mathbf{p}_{M}(0)\right\| \\
& { }^{I} \mathbf{p}_{t b x}<{ }^{I} \mathbf{p}_{t e x},
\end{array}
$$

where the coefficients $\boldsymbol{\alpha}_{5}$ and berth position ${ }^{I} \mathbf{p}_{t b}$ are variables to be optimized, and $\mathbf{X}=\left[\boldsymbol{\alpha}_{5},{ }^{I} \mathbf{p}_{t b}\right]$.

A genetic algorithm is employed to solve this minimization problem because of its fast and stochastic searching ability. Here, the designation and procedure of the trajectory planning strategy is sketched. A flowchart is shown in Figure 3.

Step 1. Coding. Choose real-number coding, and vector $\mathbf{X}$ is the individual
Step 2. Fitness function. Let the fitness function $f(\mathbf{X})$ equal the objective function $J_{z d}$ or $J_{a a}$. The smaller the value of $f(\mathbf{X})$, the better the individual

Step 3. Initialization. Set the genetic algorithm parameters, including the population size $n_{s}$, maximum generation number $n_{g}$, crossover fraction $p_{c}$, mutation fraction $p_{m}$, and stopping criterion $\varepsilon$. Then, generate the first generation randomly in terms of constraints

Step 4. Fitness calculation. Fitness functions for the two cases consist of two parts. Here, the calculation process is detailed as follows:

(a) Calculate ${ }_{C_{i}}^{C_{0}} g,{ }_{E}^{C_{0}} g$ by substituting Equation (38) into Equations (1) and (2), respectively. Thus, ${ }^{C_{0}} R_{i},{ }^{C_{0}} \mathbf{p}_{i}$ is known

(b) Denote ${ }^{I} R_{0}, J_{\text {Euler }}$ by substituting undetermined $\Psi$ into Equations (4) and (23), respectively

(c) Denote ${ }^{I} \mathbf{p}_{0}$ by substituting ${ }^{I} R_{0},{ }^{C_{0}} \mathbf{p}_{i}$ into Equation (9), where ${ }^{I} \mathbf{p}_{C}=0$

(d) Denote the generalized Jacobian matrix of the base $J_{B}$ by substituting ${ }_{C_{0}}^{I} g,{ }_{C_{i}}^{C_{0}} g$ into Equation (19)

(e) Calculate the final attitude of the base $\Psi$ by Equation (26)

(f) Calculate $J_{B \omega}$ by using Step 4 and Equation (21)

(g) Calculate $\log \left({ }_{E}^{I} g_{d}^{-1}{ }_{E} g\right)$, where ${ }_{E}^{I} g$ can be calculated by Equation (10), and ${ }_{E}^{I} g_{d}$ can be calculated by Equations (29), (30), and (44)

From the above, $f(\mathbf{X})$ can be achieved. Then, rank individuals according to their fitness values and judge whether the minimum fitness value is less than $\varepsilon$. If yes, go to Step 7; otherwise, go to the next step.

Step 5. Reproduction. Generate the next generation by the selection, crossover, and mutation operator

Selection. Use the Roulette Wheel Selection method, in which the area of each individual depends on its rank

Crossover. Choose the scatter crossover operator; a random binary vector selects the genes of the parents, which then combine to form the child

Mutation. Modify the classical Gaussian mutation method, which is usually used for unconstrained problems. The Gaussian mutation method adds random numbers from a Gaussian distribution in terms of $\left(\mu_{k}, \sigma_{k}\right)$ to the parent, and $\sigma_{k}$ shrinks as the generations increase. The method has two disadvantages: the first is that the genes of the child might exceed the boundary, and the second is that the process might slow down to find the optimal solution when 


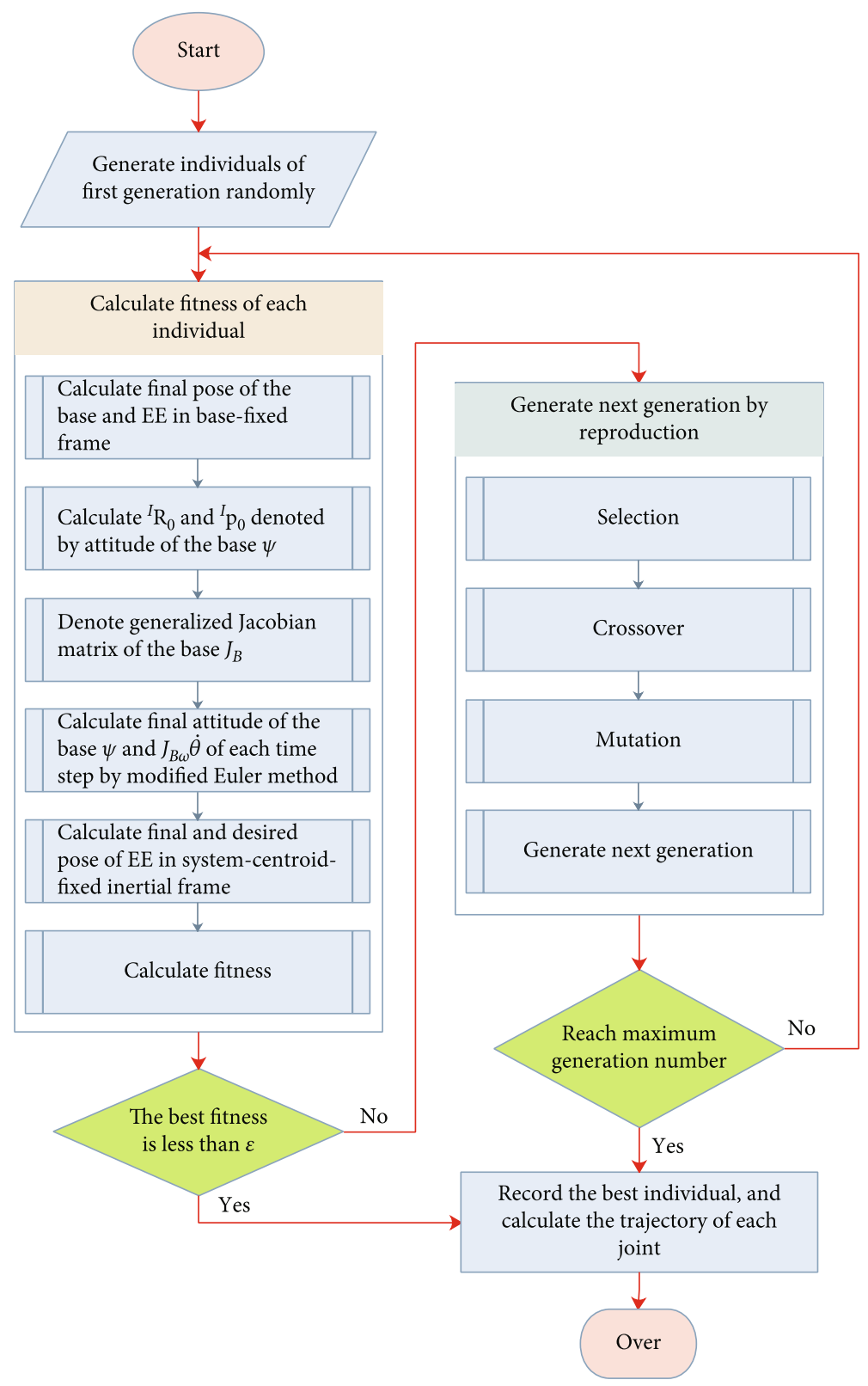

FIGURE 3: Flowchart of the provided trajectory planning strategy.

performing the local search because $\sigma_{k}$ only varies with the generation. In view of these shortcomings, we offer a modified Gaussian mutation method. The determination of $\sigma_{k}$ is defined as follows:

$$
\left\{\begin{array}{l}
\sigma_{k}=X_{\text {bound }}\left(1-\frac{k}{n_{g}}\right) \text { if } f_{\text {best }} \geq 1, \\
\sigma_{k}=X_{\text {bound }}\left(1-\frac{k}{n_{g}}\right)\left(0.5 \exp \left(f_{\text {best }}\right)-0.5\right) \text { if } 0.05<f_{\text {best }}<1, \\
\sigma_{k}=X_{\text {bound }}\left(1-\frac{k}{n_{g}}\right)\left(0.8 \exp \left(f_{\text {best }}\right)-0.8\right) \text { if } f_{\text {best }} \leq 0.05,
\end{array}\right.
$$

where $X_{\text {bound }}=X_{\max }-X_{\min }, k$ is current generation number, and $f_{\text {best }}$ is the smallest fitness value of the current population. Furthermore, correction is made when the genes of the child exceed the boundary:

$$
\left\{\begin{array}{l}
X_{i}=X_{\mathrm{maxi}}-r\left(X_{\mathrm{maxi}}-X_{\mathrm{mini}}\right) \text { if } X_{i}>X_{\mathrm{maxi}} \\
X_{i}=X_{\mathrm{mini}}+r\left(X_{\mathrm{maxi}}-X_{\mathrm{mini}}\right) \text { if } X_{i}<X_{\mathrm{mini}}
\end{array}\right.
$$

where $X_{i}$ is the $i^{\text {th }}$ gene of the child and $r$ is a random number between $(0,1)$.

Step 6. Stopping judgment. Judge whether the number of the current generation exceeds $n_{g}$. If yes, go to Step 7; otherwise, go to Step 4 


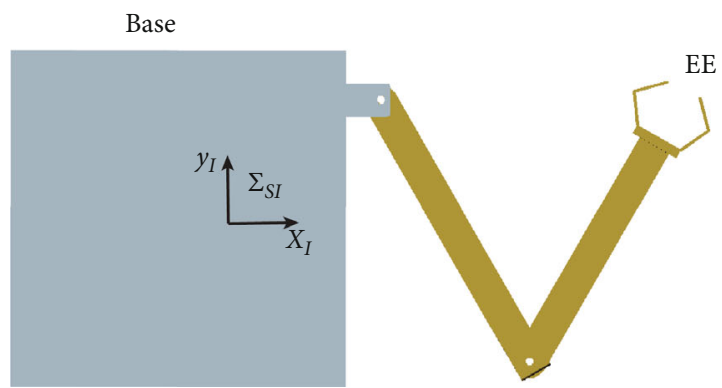

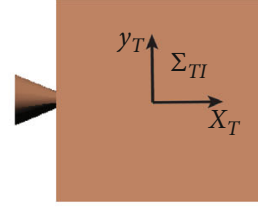

Target

FIgURE 4: Simulation model of the planar dual-link space robot in the initial configuration.

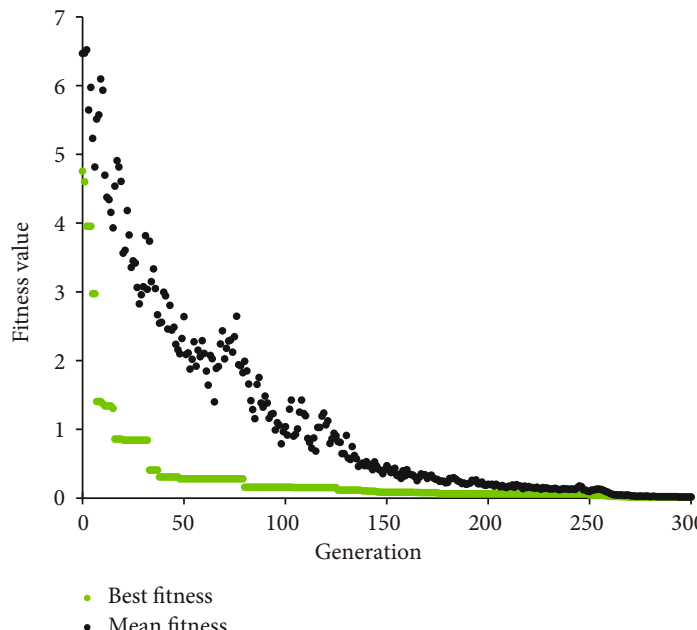

(a)

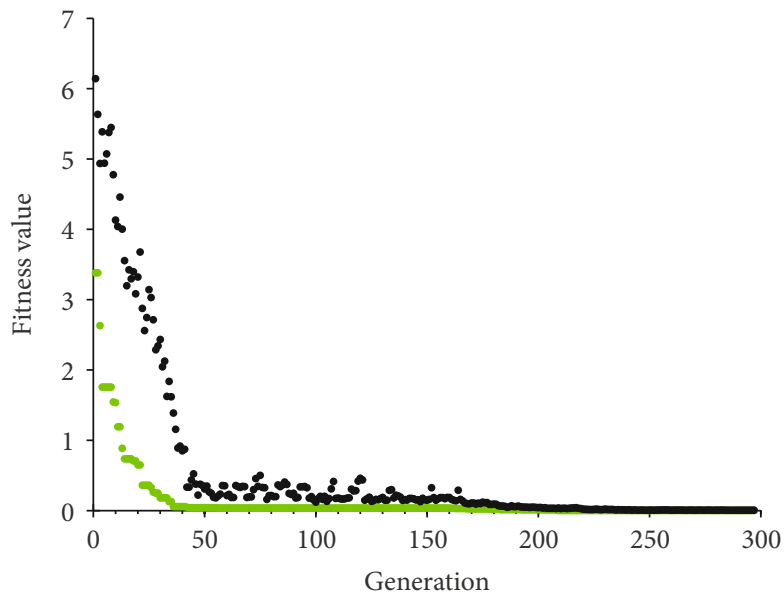

- Best fitness

- Mean fitness

(b)

Figure 5: Variation in the mean fitness and best fitness of each generation.

Step 7. Trajectory calculation. Obtain the population containing the best individual. Calculate the trajectory of each joint using $a_{i 5}$ of the best individual. Thus, the planning concludes

\section{Simulation Examples}

Trajectory planning of a planar dual-link space robot was performed with MATLAB. To verify the effectiveness of our strategy and illustrate berth position and the moving process of optimized joint trajectory, we built a corresponding model by Adams. The optimal results of each calculation were substituted into the Adams model, and Adams simulations were run. The simulation model in the initial configuration is shown in Figure 4. The CPU clock of the computer used is $3.5 \mathrm{GHz}$.

The detailed initial and inertia parameters of the model are as follows.

$$
\begin{aligned}
& \boldsymbol{\omega}_{1}=\boldsymbol{\omega}_{2}=(0,0,1)^{\mathrm{T}}, \mathbf{r}_{1}=(1.2,0.7,0)^{\mathrm{T}}, \mathbf{r}_{2}=(2.09,-0.85,0)^{\mathrm{T}} . \\
& \text { Then, } \boldsymbol{\xi}_{1}=\left(\boldsymbol{\omega}_{1}, \mathbf{r}_{1} \times \boldsymbol{\omega}_{1}\right)^{\mathrm{T}}=(0,0,1,0.7,-1.2,0)^{\mathrm{T}}, \boldsymbol{\xi}_{2}=\left(\boldsymbol{\omega}_{2},\right. \\
& \left.\mathbf{r}_{2} \times \boldsymbol{\omega}_{2}\right)^{\mathrm{T}}=(0,-1,0,-0.85,-2.09,0)^{\mathrm{T}} .
\end{aligned}
$$
7.8 ;

Next, $m_{0}=400, m_{1}=18, m_{2}=24 ; I_{z 0}=260, I_{z 1}=6, I_{z 2}=$

$$
\begin{aligned}
& { }_{C_{1}}^{B} g(0)=\left[\begin{array}{cc}
R_{z}\left(-60^{\circ}\right) & -0.14 \\
0 & 0 \\
0 & 1
\end{array}\right], \\
& { }_{C_{2}}^{B} g(0)=\left[\begin{array}{cc}
R_{z}\left(60^{\circ}\right) & 0.12 \\
0 & 0 \\
0 & 1
\end{array}\right], \\
& { }_{E}^{B} g(0)=\left[\begin{array}{cc}
3.65 \\
R_{z}\left(60^{\circ}\right) & 0.72 \\
0 & 0 \\
0 & 1
\end{array}\right],
\end{aligned}
$$



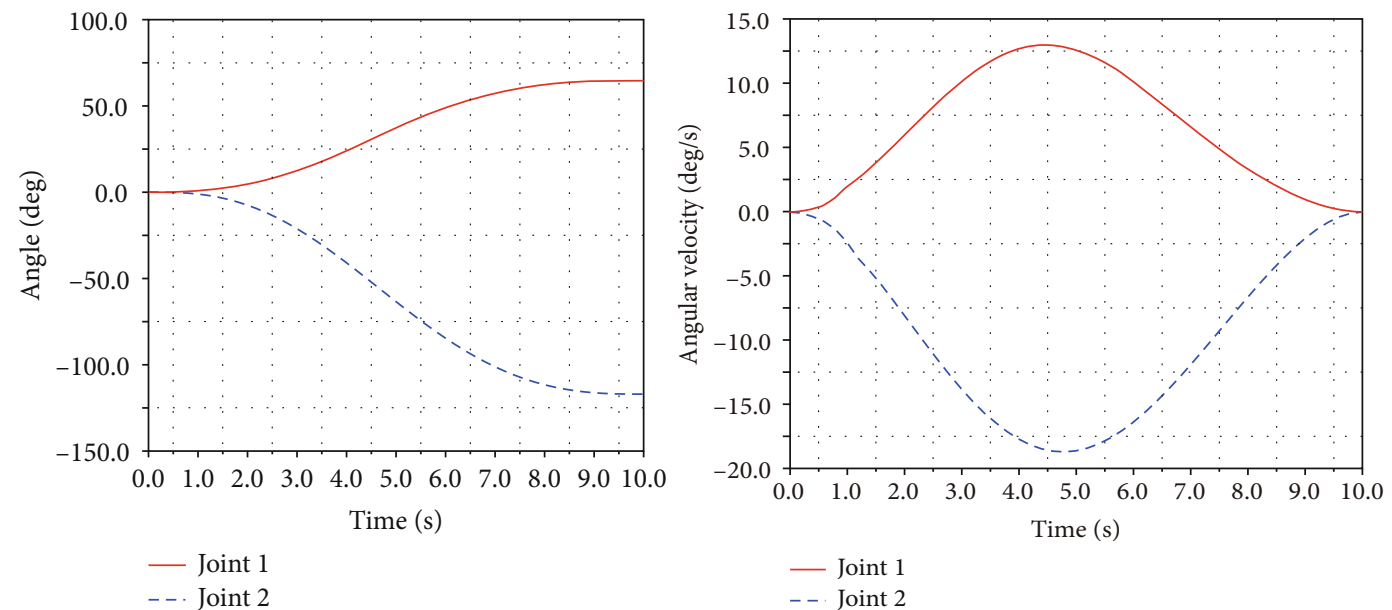

FIGURE 6: Optimized trajectories of joints for the planning case of zero attitude disturbance.
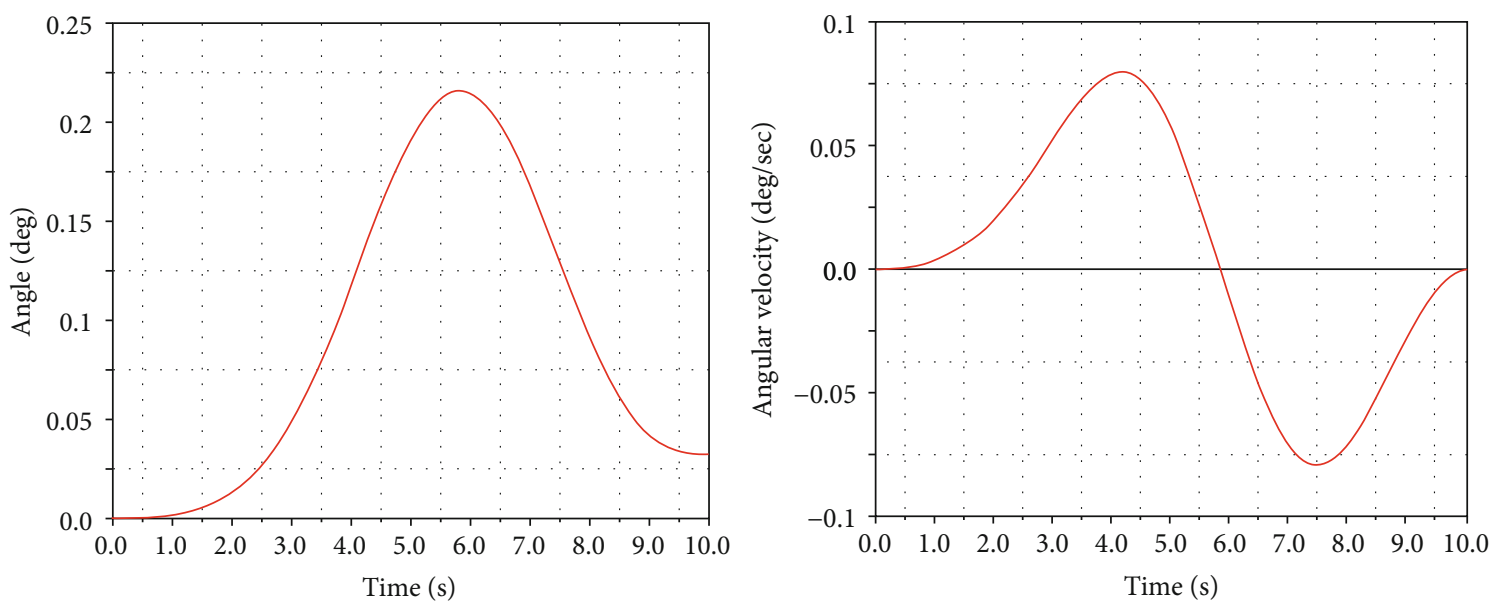

FIGURE 7: Optimized trajectory of the base for the planning case of zero attitude disturbance.

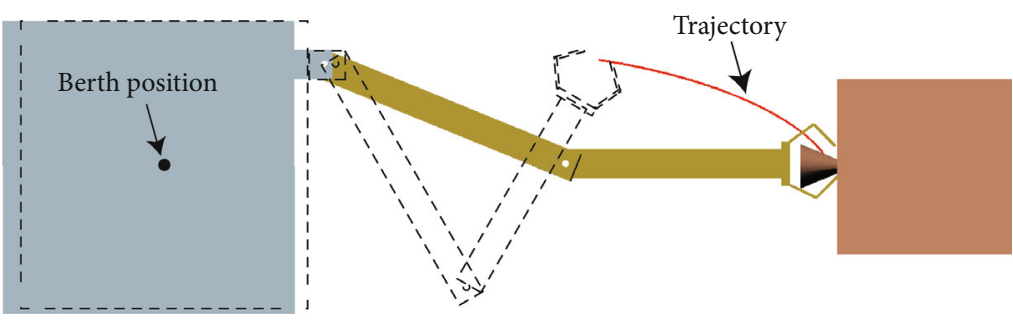

FiguRE 8: Optimized berth position and motion process for the planning case of zero attitude disturbance.

where

$$
R_{z}(\phi)=\left[\begin{array}{ccc}
\cos \phi & -\sin \phi & 0 \\
\sin \phi & \cos \phi & 0 \\
0 & 0 & 1
\end{array}\right]
$$

is the direction cosine matrix along $z$ axis. According to Equation $(27), \mathbf{p}_{M}(0)=(-0.212,0,0)^{\mathrm{T}}$ and ${ }^{\mathrm{I}} \mathbf{p}_{t e}=(-0.65,0,0)^{\mathrm{T}}$,
$P_{s}=3.60, R_{w}=4.69$, so constraints of ${ }^{I} \mathbf{p}_{t b}$ can be given as $3.60<\left\|{ }^{I} \mathbf{p}_{t b}\right\|<5.55$ and ${ }^{I} \mathbf{p}_{t b x}<-0.65$. Constraints of the angular variables are as follows.

$$
\begin{cases}\boldsymbol{\theta}_{\min }=(-75,-165)^{\mathrm{T}}\left(^{\circ}\right), & \boldsymbol{\theta}_{\max }=(75,165)^{\mathrm{T}}\left(^{\circ}\right), \\ \dot{\boldsymbol{\theta}}_{\text {min }}=(-30,-35)^{\mathrm{T}}(\% / \mathrm{s}), & \dot{\boldsymbol{\theta}}_{\max }=(30,35)^{\mathrm{T}}\left({ }^{\circ} / \mathrm{s}\right), \quad . \\ \ddot{\boldsymbol{\theta}}_{\text {min }}=(-15,-20)^{\mathrm{T}}\left({ }^{\circ} / \mathrm{s}^{2}\right), & \ddot{\boldsymbol{\theta}}_{\max }=(15,20)^{\mathrm{T}}\left({ }^{\circ} / \mathrm{s}^{2}\right) .\end{cases}
$$



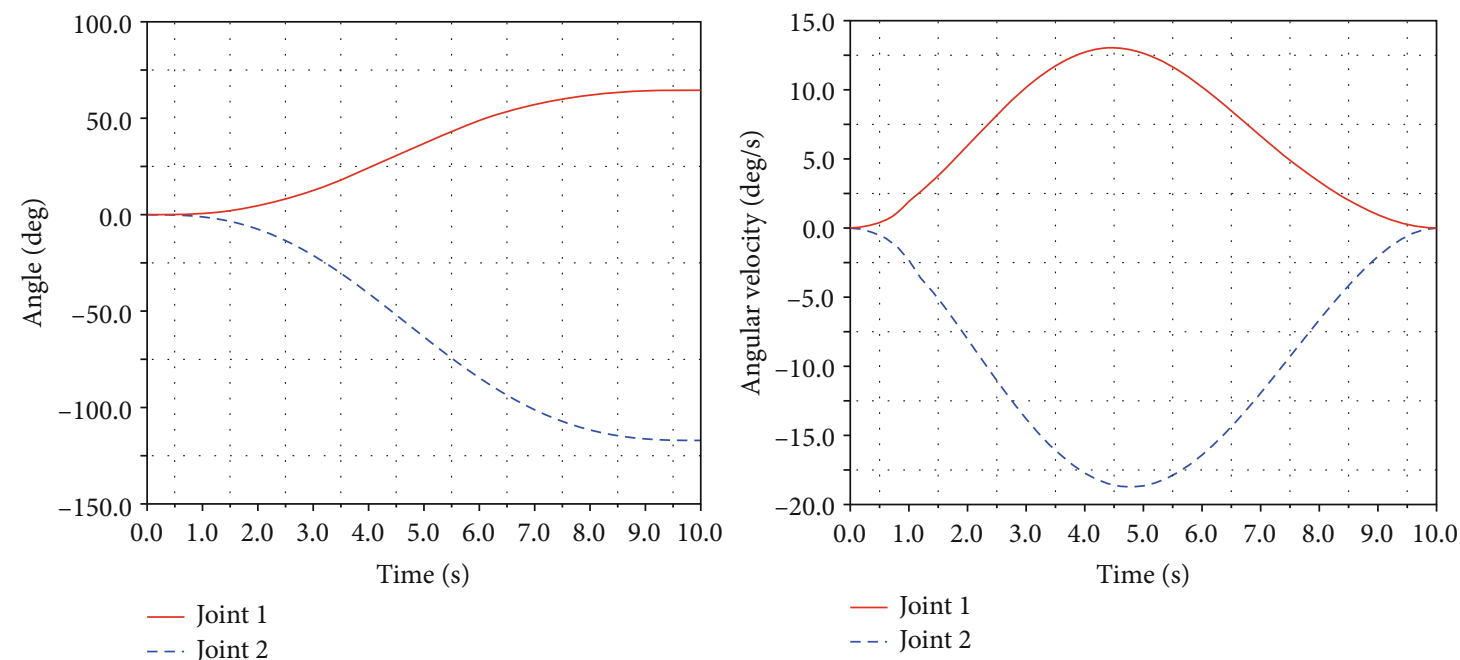

Figure 9: Optimized trajectories of joints for the planning case of attitude adjustment.
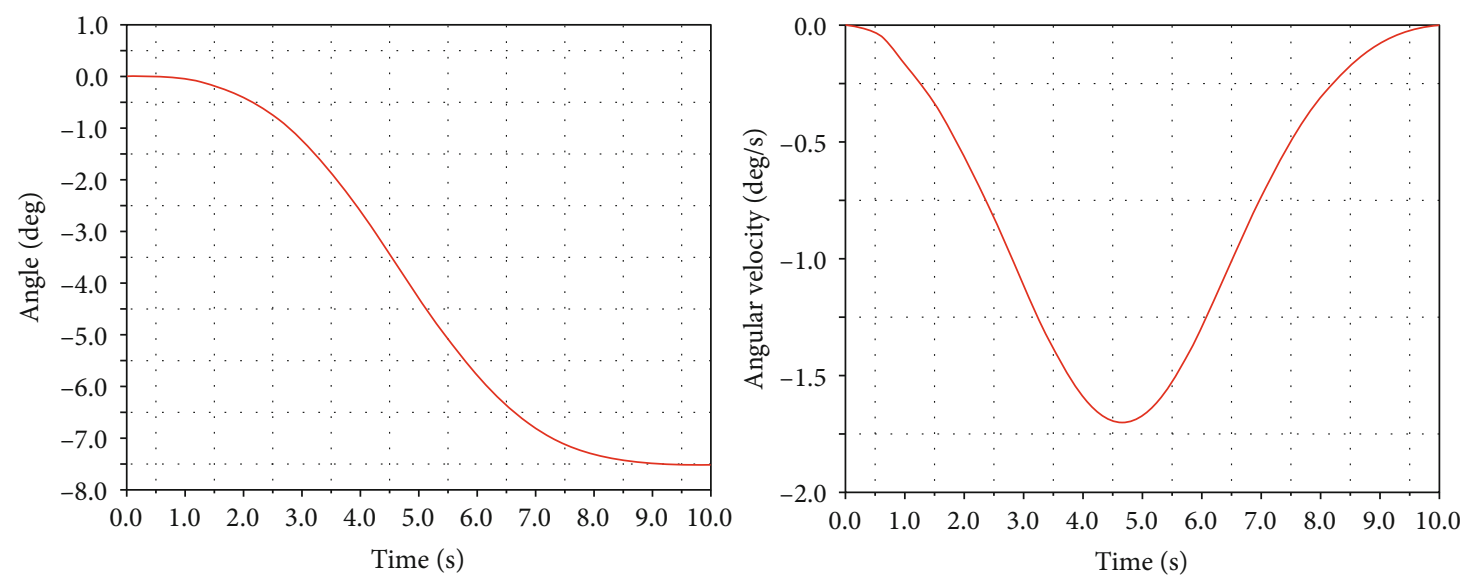

FIgURE 10: Optimized trajectory of the base for the planning case of attitude adjustment.

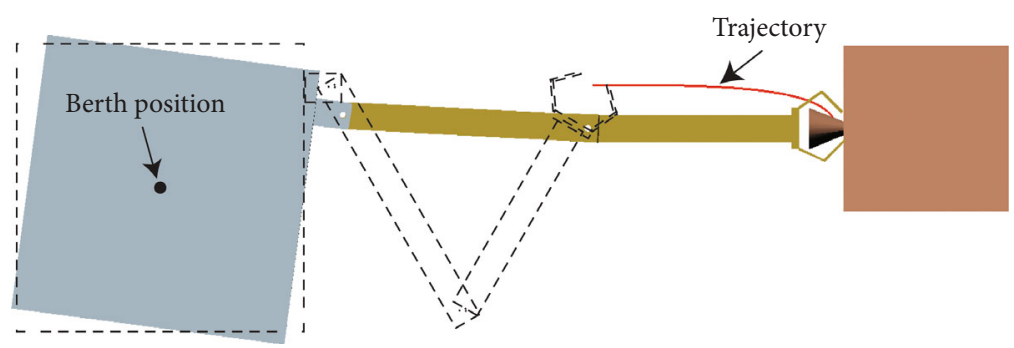

FIgURE 11: Optimized berth position and motion process for the planning case of attitude adjustment.

According to Equations (42) and (43), $\boldsymbol{\alpha}_{5 \min }=$ $(-2.002,-0.801)^{\mathrm{T}} \times 10^{-4}, \boldsymbol{\alpha}_{5 \max }=(2.002,0.801)^{\mathrm{T}} \times 10^{-4}, l_{1}=$ $(75,165)^{\mathrm{T}}, l_{2}=(0,0)^{\mathrm{T}}$, where $t_{f}=10.0 \mathrm{~s}$.

The desired orientation of the EE is set at ${ }_{E}^{P} R_{d}=R_{z}\left(-90^{\circ}\right)$, and the desired attitude of the base is set as $\Psi_{d}=\left[0,0,-7.5^{\circ}\right]$ for attitude adjustment. The parameters of the genetic algorithm are set at $n_{s}=45, n_{g}=300, p_{c}=0.83, p_{m}=0.08, \varepsilon=$ 0.001 . The results of the simulation using MATLAB programs are as follows.
For the planning case of zero attitude disturbance to the base, the iteration stops when the maximum generation number is reached. The variation in the mean fitness and best fitness of each generation is shown in Figure 5(a). The optimal individual is $\mathbf{X}_{\text {best }}=[-5.227,-0.010,0.316,-0.380]$, and the corresponding fitness is $f\left(\mathbf{X}_{\text {best }}\right)=0.0144$. The disturbance to the base attitude is tolerable, and the maximum amplitudes of the base's angle and angular velocity are $0.216^{\circ}$ and $0.080^{\circ} / \mathrm{s}$, respectively. The optimized trajectories of the joints and the base attitude are shown in Figures 6 
and 7 , respectively. Figure 8 shows the optimized berth position and motion process simulated by Adams, with the dotted lines denoting the initial configuration. It can be observed that the EE reaches the capture point precisely, and the base attitude is nearly changeless. The trajectory of the EE is also sketched. The calculation time for each simulation is about $7.5 \mathrm{~min}$.

For the planning case of attitude adjustment of the base, iteration stops at the $297^{\text {th }}$ generation as the stopping criterion $\varepsilon$ is satisfied. Variation in the mean fitness and the best fitness of each generation is shown in Figure 5(b). The optimal individual is $\mathbf{X}_{\text {best }}=[-5.422,-0.401,0.622,-0.473]$, and the corresponding fitness is $f\left(\mathbf{X}_{\text {best }}\right)=0.00087$. The final attitude of the base achieves the desired adjustment, $\Psi_{f}=[0,0$, $\left.-7.514^{\circ}\right]$, and the corresponding error is $0.19 \%$ compared with $\Psi_{d}$. The optimized trajectories of the joints and the base attitude are shown in Figures 9 and 10, respectively. Figure 11 shows the optimized berth position and motion process simulated by Adams, and it shows that the EE again reaches the capture point precisely. The trajectory of the $\mathrm{EE}$ is also sketched. The calculation time for each simulation is about $8 \mathrm{~min}$.

\section{Conclusion}

In this paper, several innovations are presented to improve the approach to the trajectory planning of a free-floating space robot. First, there are two useful techniques to improve the calculation efficiency: systematical deduction of the generalized Jacobian matrix using screw theory and the calculation of the base attitude by the modified Euler method without integration. Second, the berth position is optimized with the joint variables by the transformation between the target-centroid-fixed and system-centroid-fixed inertial frame. The extra goals proposed are all achieved by considering the berth position, while the EE reaches the desired position and orientation. More importantly, the simulations of example missions are completed by using only two joints. Figure 5 shows that the presented optimization method converges quickly and stably, thereby demonstrating that the proposed objective function is of good quality. Furthermore, the optimized trajectories of both the joints and the EE are feasible and smooth.

\section{Nomenclature}

\begin{tabular}{|c|c|}
\hline$\sum_{I}:$ & The general inertial frame \\
\hline$\Sigma_{S I}, \Sigma_{T I}:$ & $\begin{array}{l}\text { System-centroid-fixed, target-centroid-fixed } \\
\text { inertial frame }\end{array}$ \\
\hline$\Sigma_{C I}$ & $\begin{array}{l}\text { The centroid-fixed frame of the } i^{t h} \operatorname{link}(i=0,1 \text {, } \\
\ldots, n, 0 \text { represents the base hereinafter })\end{array}$ \\
\hline & The end-effector-fixed frame \\
\hline & $\begin{array}{l}\text { The position of the centroid of the } i^{\text {th }} \text { link } \\
\text { expressed in frame } A \text { ( } A \text { denotes an arbitrary } \\
\text { frame hereinafter) }\end{array}$ \\
\hline & $\begin{array}{l}\text { The position of the end-effector expressed in } \\
\text { frame } A\end{array}$ \\
\hline & $\begin{array}{l}\text { The position of the centroid of the system } \\
\text { expressed in frame } A\end{array}$ \\
\hline
\end{tabular}

${ }^{A} R_{i}: \quad$ The orientation matrix of the centroid of the $i^{t h}$ link expressed in frame $A$

${ }^{A} R_{E}: \quad$ The orientation matrix of the end-effector expressed in frame $A$

${ }_{A}^{B} g$ : $\quad$ The pose of frame $A$ expressed in frame $B$, and ${ }_{A}^{B} g=\left[\begin{array}{cc}{ }_{A}^{B} R & { }^{B} p_{A} \\ 0 & 1\end{array}\right]$

$\theta_{i}: \quad$ The angle of joint $i$

$\omega_{i}$ : $\quad$ The rotational axis of joint $i$

$\boldsymbol{r}_{i}$ : $\quad$ An arbitrary point on the rotational axis of joint $i$

$\widehat{\xi}_{i} \in \operatorname{se}(3):$ The corresponding twist of joint $i$

$e^{\theta_{i} \widehat{\xi}_{i}}: \quad$ The matrix exponential of $\theta_{i} \widehat{\xi}_{i}$

$I_{C_{i}}$ : The inertial tensor of the $i^{\text {th }}$ link with respect to its body frame

$m_{i}$ : The mass of the $i^{\text {th }}$ link

$\theta$ : $\quad$ The operator that maps a vector to the corresponding skew-symmetric matrix (or twist), $\mathbb{R}^{3}$ $\rightarrow$ so $(3)$

()$^{\vee}: \quad$ The operator that maps a skew-symmetric matrix (or twist) to the corresponding vector, so(3) $\rightarrow$ $\mathbb{R}^{3}$.

\section{Data Availability}

The data used to support the findings of this study are included within the article.

\section{Conflicts of Interest}

The authors declare that there is no conflict of interest regarding the publication of this paper.

\section{Acknowledgments}

This research is funded by the Shanghai Aerospace Science and Technology Innovation Foundation (Grant No. SAST2017-020).

\section{References}

[1] J. N. Pelton, On-Orbit Servicing and Retrofitting, Springer, New York, NY, USA, 2016.

[2] A. Ellery, J. Kreisel, and B. Sommer, "The case for robotic onorbit servicing of spacecraft: spacecraft reliability is a myth," Acta Astronautica, vol. 63, no. 5-6, pp. 632-648, 2008.

[3] C. Sallaberger, Space Plan Task Force, and Canadian Space Agency, "Canadian space robotic activities," Acta Astronautica, vol. 41, no. 4-10, pp. 239-246, 1997.

[4] M. Oda, K. Kibe, and F. Yamagata, "Ets-vii, space robot inorbit experiment satellite," in Proceedings of IEEE International Conference on Robotics and Automation, pp. 739-744, Minneapolis, MN, USA, April 1996.

[5] T. Debus and S. Dougherty, "Overview and performance of the front-end robotics enabling near-term demonstration (frend) robotic arm," in AIAA Infotech@Aerospace Conference, pp. 1-12, Seattle, WA, USA, April 2009.

[6] Y. Hu, Y. Xue, Y. Yue, and X. Shi, "China's intelligent space robot enters world advanced level," Aerospace China, vol. 16, no. 4, pp. 29-34, 2015. 
[7] P. Boning and S. Dubowsky, "Coordinated control of space robot teams for the on-orbit construction of large flexible space structures," Advanced Robotics, vol. 24, no. 3, pp. 303323, 2010.

[8] G. Rekleitis and E. Papadopoulos, "On-orbit cooperating space robotic servicers handling a passive object," IEEE Transactions on Aerospace and Electronic Systems, vol. 51, no. 2, pp. 802814, 2015.

[9] L. Jackson, C. Saaj, A. Seddaoui, C. Whiting, S. Eckersley, and M. Ferris, "Design of a small space robot for on-orbit assembly missions," in ICMRE'19 Proceedings of the 5th International Conference on Mechatronics and Robotics Engineering, pp. 107-112, Rome, Italy, February 2019.

[10] A. Flores-Abad, O. Ma, K. Pham, and S. Ulrich, "A review of space robotics technologies for on-orbit servicing," Progress in Aerospace Sciences, vol. 68, pp. 1-26, 2014.

[11] S. Dubowsky and E. Papadopoulos, "The kinematics, dynamics, and control of free-flying and free-floating space robotic systems," IEEE Transactions on Robotics and Automation, vol. 9, no. 5, pp. 531-543, 1993.

[12] E. Papadopoulos and S. Dubowsky, "Dynamic singularities in free-floating space manipulators," in Space Robotics: Dynamics and Control. The Kluwer International Series in Engineering and Computer Science, vol 188, Y. Xu and T. Kanade, Eds., pp. 77-100, Springer, Boston, MA, USA, 1993.

[13] W. Xu, C. Li, X. Wang, Y. Liu, B. Liang, and Y. Xu, "Study on non-holonomic Cartesian path planning of a free-floating space robotic system," Advanced Robotics, vol. 23, no. 1-2, pp. 113-143, 2009.

[14] Z. Vafa and S. Dubowsky, "The kinematics and dynamics of space manipulators: the virtual manipulator approach," The International Journal of Robotics Research, vol. 9, no. 4, pp. 3-21, 1990.

[15] Z. Vafa and S. Dubowsky, "On the dynamics of space manipulators using the virtual manipulator, with applications to path planning," The Journal of the Astronautical Sciences, vol. 38, no. 4, pp. 441-472, 1990.

[16] S. Dubowsky and M. A. Torres, "Path planning for space manipulators to minimize spacecraft attitude disturbances," in Proceedings. 1991 IEEE International Conference on Robotics and Automation, pp. 2522-2528, Sacramento, CA, USA, April 1991.

[17] M. A. Torres and S. Dubowsky, "Minimizing spacecraft attitude disturbances in space manipulator systems," Journal of Guidance, Control, and Dynamics, vol. 15, no. 4, pp. 10101017, 1992.

[18] C. Fernandes, L. Gurvits, and Z. Li, "Near-optimal nonholonomic motion planning for a system of coupled rigid bodies," IEEE Transactions on Automatic Control, vol. 39, no. 3, pp. 450-463, 1994.

[19] E. Papadopoulos, I. Tortopidis, and K. Nanos, "Smooth planning for free-floating space robots using polynomials," in Proceedings of the 2005 IEEE International Conference on Robotics and Automation, pp. 4272-4277, Barcelona, Spain, April 2005.

[20] I. Tortopidis and E. Papadopoulos, "On point-to-point motion planning for underactuated space manipulator systems," Robotics and Autonomous Systems, vol. 55, no. 2, pp. 122131, 2007.

[21] W.-F. Xu, X.-Q. Wang, Q. Xue, and B. Liang, "Study on trajectory planning of dual-arm space robot keeping the base stabilized," Acta Automatica Sinica, vol. 39, no. 1, pp. 69-80, 2013.
[22] P. Huang, Y. Xu, and B. Liang, "Global minimum-jerk trajectory planning of space manipulator," International Journal of Control, Automation, and Systems, vol. 4, no. 4, pp. 405-413, 2006.

[23] W. Xu, Y. Liu, B. Liang, Y. Xu, C. Li, and W. Qiang, "Nonholonomic path planning of a free-floating space robotic system using genetic algorithms," Advanced Robotics, vol. 22, no. 4, pp. 451-476, 2008.

[24] W. Xu, C. Li, B. Liang, Y. Liu, and Y. Xu, "The Cartesian path planning of free-floating space robot using particle swarm optimization," International Journal of Advanced Robotic Systems, vol. 5, no. 3, p. 27, 2008.

[25] M. Wang, J. Luo, and U. Walter, "Trajectory planning of freefloating space robot using particle swarm optimization (PSO)," Acta Astronautica, vol. 112, pp. 77-88, 2015.

[26] M. Wang, J. Luo, J. Yuan, and U. Walter, "Coordinated trajectory planning of dual-arm space robot using constrained particle swarm optimization," Acta Astronautica, vol. 146, pp. 259-272, 2018.

[27] T. Rybus and K. Seweryn, "Application of rapidly-exploring random trees (RRT) algorithm for trajectory planning of free-floating space manipulator," in 2015 10th International Workshop on Robot Motion and Control (RoMoCo), pp. 9196, Poznan, Poland, July 2015.

[28] J. Luo, M. Yu, M. Wang, and J. Yuan, "A fast trajectory planning framework with task-priority for space robot," Acta Astronautica, vol. 152, pp. 823-835, 2018.

[29] X. Zhang and J. Liu, "Effective motion planning strategy for space robot capturing targets under consideration of the berth position," Acta Astronautica, vol. 148, pp. 403-416, 2018.

[30] Y. Umetani and K. Yoshida, "Continuous path control of space manipulators mounted on OMV," Acta Astronautica, vol. 15, no. 12, pp. 981-986, 1987.

[31] B. Liang, Y. Xu, and M. Bergerman, "Mapping a space manipulator to a dynamically equivalent manipulator," Journal of Dynamic Systems, Measurement, and Control, vol. 120, pp. 1-7, 1998.

[32] K. Yoshida and Y. Umetani, "Control of space manipulators with generalized Jacobian matrix," in Space Robotics: Dynamics and Control. The Kluwer International Series in Engineering and Computer Science, vol 188, Y. Xu and T. Kanade, Eds., pp. 165-204, Springer, Boston, MA, USA, 2011.

[33] A. Pisculli, L. Felicetti, P. Gasbarri, and G. Palmerini, "A reaction-null/Jacobian transpose control strategy with gravity gradient compensation for on-orbit space manipulators," Aerospace Science and Technology, vol. 38, pp. 3040, 2014.

[34] X. Zhang and J. Liu, "Autonomous trajectory planner for space telerobots capturing space debris under the teleprogramming framework," Advances in Mechanical Engineering, vol. 9, no. 9, 13 pages, 2017.

[35] E. Sariyildiz, E. Cakiray, and H. Temeltas, "A comparative study of three inverse kinematic methods of serial industrial robot manipulators in the screw theory framework," International Journal of Advanced Robotic Systems, vol. 8, no. 5, p. 64, 2011.

[36] R. Murray, L. Zexiang, and S. Sastry, A Mathematical Introduction to Robotics Manipulation, CRC press, Boca Raton, FL, USA, 1994.

[37] J. Gallardo, J. Rico, A. Frisoli, D. Checcacci, and M. Bergamasco, "Dynamics of parallel manipulators by means 
of screw theory," Mechanism and Machine Theory, vol. 38, no. 11, pp. 1113-1131, 2003.

[38] K. Ibrahim, A. A. Ramadan, M. Fanni, Y. Kobayashi, A. A. A. Ismail, and M. G. Fujie, "Kinematic analysis and control of limited 4-DOF parallel manipulator based on screw theory," in 2012 IEEE International Conference on Systems, Man, and Cybernetics (SMC), pp. 47-52, Seoul, South Korea, October 2012.

[39] C. Rocha, C. Tonetto, and A. Dias, "A comparison between the Denavit-Hartenberg and the screw-based methods used in kinematic modeling of robot manipulators," Robotics and Computer-Integrated Manufacturing, vol. 27, no. 4, pp. 723728,2011

[40] J. Denavit and R. Hartenberg, "A kinematic notation for lowerpair mechanisms," Journal of Applied Mechanics, vol. 22, no. 2, pp. 215-221, 1955.

[41] F. Liu and H. Wu, "Research on modeling of robot manipulator dynamics based on screw theory," Journal of Jiangsu University of Science and Technology (Natural Science Edition), vol. 22, no. 2, pp. 52-55, 2008.

[42] Y. Liu and H. Wu, "Deduction of Jacobian matrix for freefloating branching space robot based on recursion of screw," China Mechanical Engineering, vol. 21, no. 11, pp. 12751277, 2010.

[43] Y. Wang, X. Liang, K. Gong, and Y. Liao, "Kinematical research of free-floating space-robot system at position level based on screw theory," International Journal of Aerospace Engineering, vol. 2019, Article ID 6857106, 13 pages, 2019. 


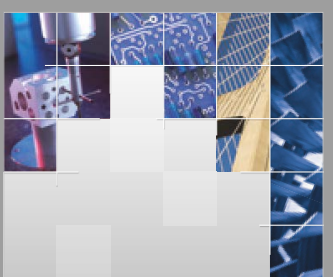

\section{Enfincering}
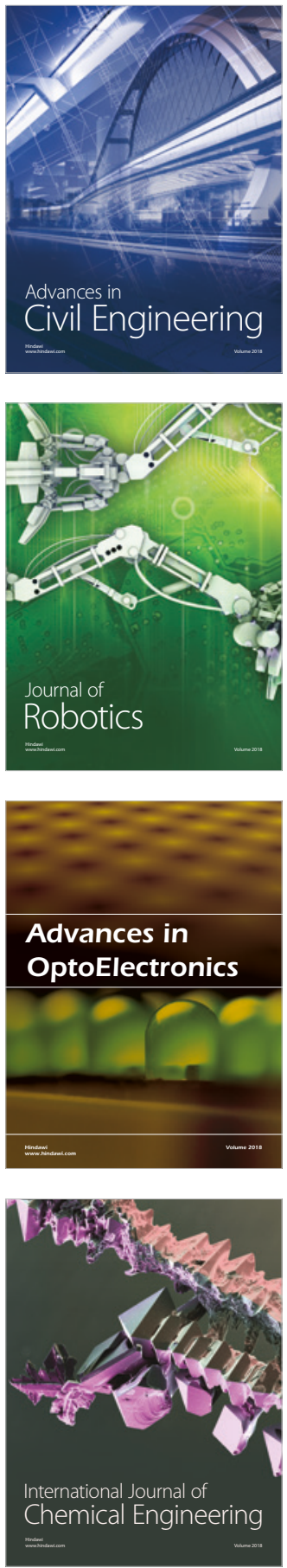

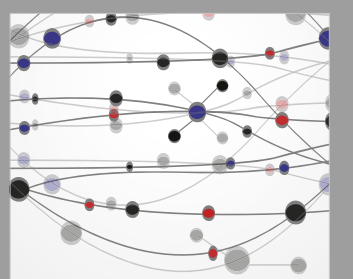

\section{Rotating \\ Machinery}

The Scientific World Journal

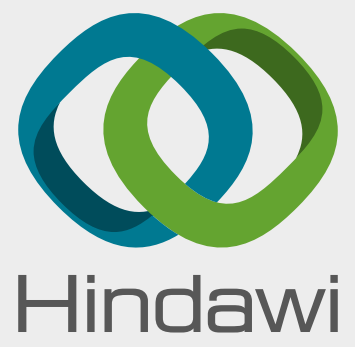

Submit your manuscripts at

www.hindawi.com
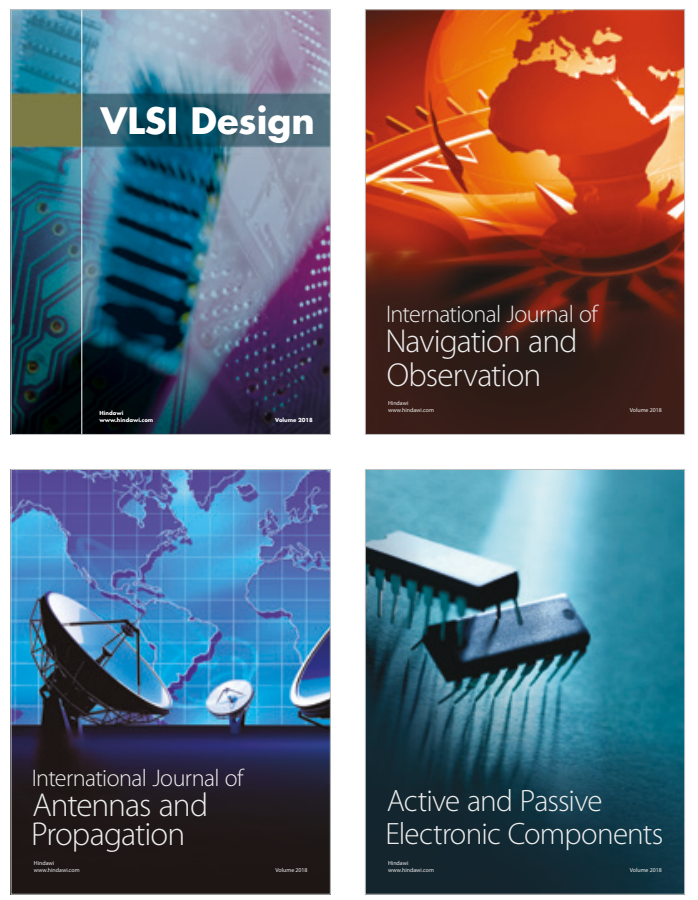
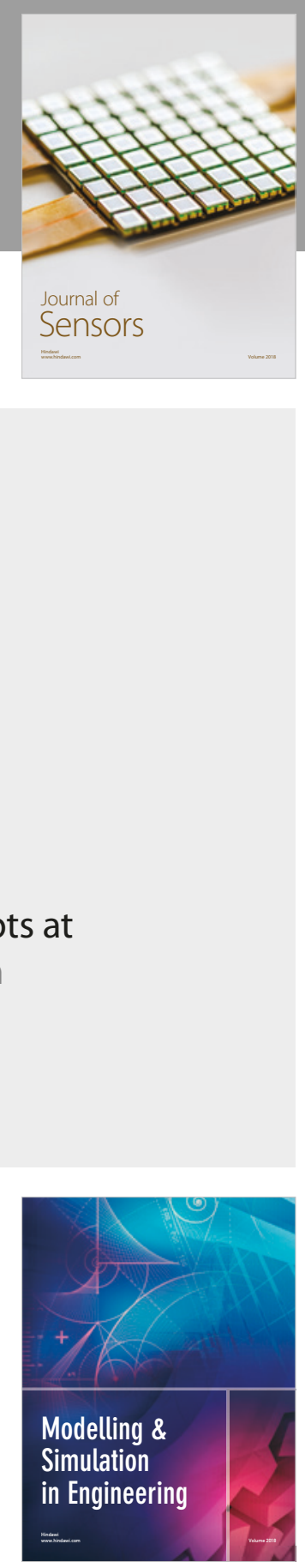

\section{Advances \\ Multimedia}
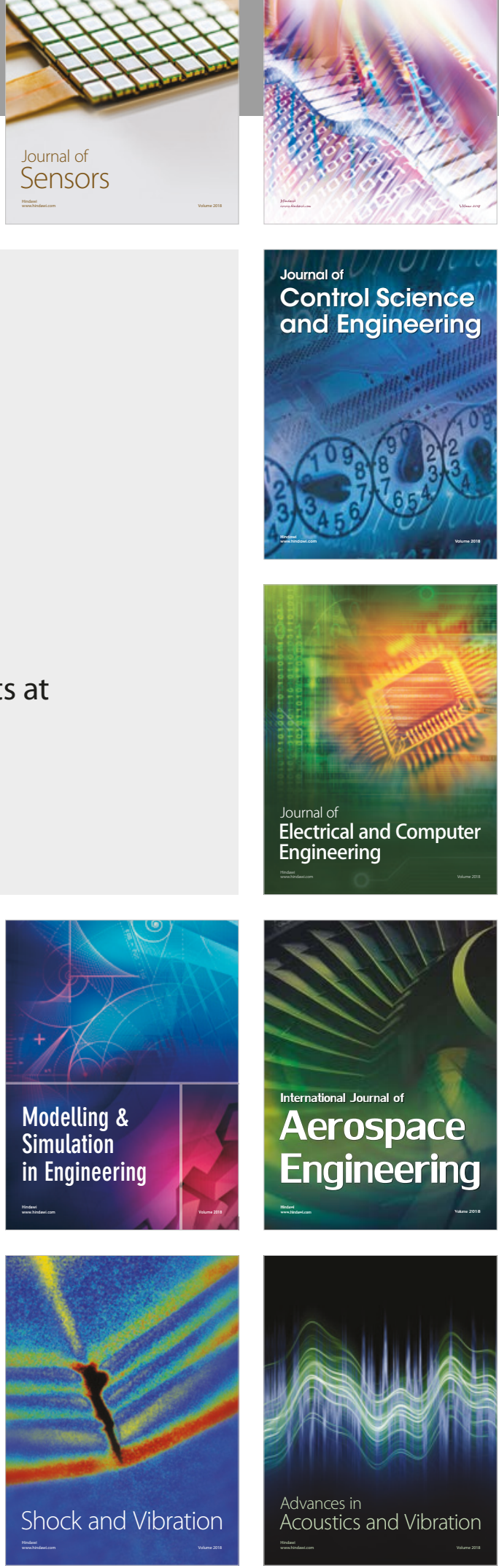